\title{
Simulation of Subsonic and Supersonic Flows in Inductive Plasmatrons
}

\author{
S. V. Utyuzhnikov, ${ }^{*}$ A. V. Konyukhov, ${ }^{\dagger}$ and D. V. Rudenko \\ Moscow Institute of Physics and Technology, 141700, Dolgoprudny, Russia \\ S. A. Vasil'evskii ${ }^{\S}$ and A. F. Kolesnikov"I \\ Russian Academy of Sciences, 119526, Moscow, Russia \\ and \\ O. Chazot** \\ von Kármán Institute for Fluid Dynamics, B-1640 Rhode Saint Genese, Belgium
}

\begin{abstract}
Simulation of sub- and supersonic thermochemical equilibrium flows in plasmatrons is considered. A physicochemical model, numerical method, and computation results for equilibrium inductive coupled plasma flows in a plasmatron are given. An effective preconditioning technique along with an implicit total-variation-diminishing scheme is used to solve the Navier-Stokes equations in both subsonic and supersonic regimes. The governing equations include source terms corresponding to the electromagnetic field influence: the Lorentz force components (so-called magnetic pressure) and Joule heat production. The necessary transport coefficients were calculated in advance for equilibrium air plasma as the functions of pressure and temperature. Transport properties were calculated by the precise formulas of the Chapman-Enskog method in the temperature range $300 \leq T \leq 15,000 \mathrm{~K}$. Calculations of equilibrium air plasma flows for the IPG-4 (Institute for Problems in Mechanics, Russian Academy of Science) discharge channel geometry with the channel radius $R_{c}=0.04 \mathrm{~m}$ and length $Z_{c}=0.40 \mathrm{~m}$ were performed. Creation of both underexpanded and overexpanded jets exhausted from the plasmatron channel is considered. A comparison with experimental results is given.
\end{abstract}

\section{Nomenclature}

$A$
$a$
$a$
$\tilde{a}^{l}$
$d_{c}$
$E$
$E$

$=$ Jacobi matrix

$a=$ sound velocity, $\mathrm{m} / \mathrm{s}$

$a^{l} \quad=$ eigenvalues of matrix $A$

$\tilde{a}^{l} \quad=$ eigenvalues of matrix $P_{c} A$

$d_{c} \quad=$ sonic nozzle diameter, $\mathrm{m}$

$E \quad=$ total energy per unit volume, $\mathrm{J} / \mathrm{m}^{3}$

$\boldsymbol{E}=$ flux vector, axial component

$E_{\theta} \quad=$ complex amplitude of electric field, tangential component, $\mathrm{V} / \mathrm{m}$

$e=$ internal energy, $\mathrm{J} / \mathrm{kg}$

$\boldsymbol{F}_{n} \quad=$ flux vector normal to the cell face

$\boldsymbol{G}=$ flux vector, radial component

$H_{z} \quad=$ complex amplitude of magnetic field, axial component, $\mathrm{A} / \mathrm{m}$

$h \quad=$ enthalpy, $\mathrm{J} / \mathrm{kg}$

$I \quad=$ unit matrix

$M \quad=$ Mach number

$\boldsymbol{n}=$ external normal vector to a cell face

$n_{x}, n_{y}=$ axial and radial components of a vector $\boldsymbol{n}$

$P_{0} \quad=$ pressure at an inlet slot, $\mathrm{hPa}$

Received 13 March 2003; presented as Paper 2003-3866 at the AIAA 34th Plasmadynamics and Lasers Conference, Orlando, FL, 23-26 June 2003; revision received 19 November 2003; accepted for publication 19 November 2003. Copyright (C) 2004 by the American Institute of Aeronautics and Astronautics, Inc. All rights reserved. Copies of this paper may be made for personal or internal use, on condition that the copier pay the $\$ 10.00$ per-copy fee to the Copyright Clearance Center, Inc., 222 Rosewood Drive, Danvers, MA 01923; include the code 0001-1452/04 \$10.00 in correspondence with the CCC.

*Professor; currently Visiting Professor, Department of Aerospace, Mechanical and Manufacturing Engineering, UMIST, Manchester, England M60 1QD, United Kingdom; s.utyuzhnikov@umist.ac.uk.

${ }^{\dagger}$ Researcher.

${ }^{\ddagger}$ Assistant Professor.

${ }^{\S}$ Senior Researcher, Institute for Problems in Mechanics, Prospect Vernadskogo 101/1.

"Leading Researcher, Institute for Problems in Mechanics, Prospect Vernadskogo 101/1

**Assistant Professor, Chaussee de Waterloo, 72.

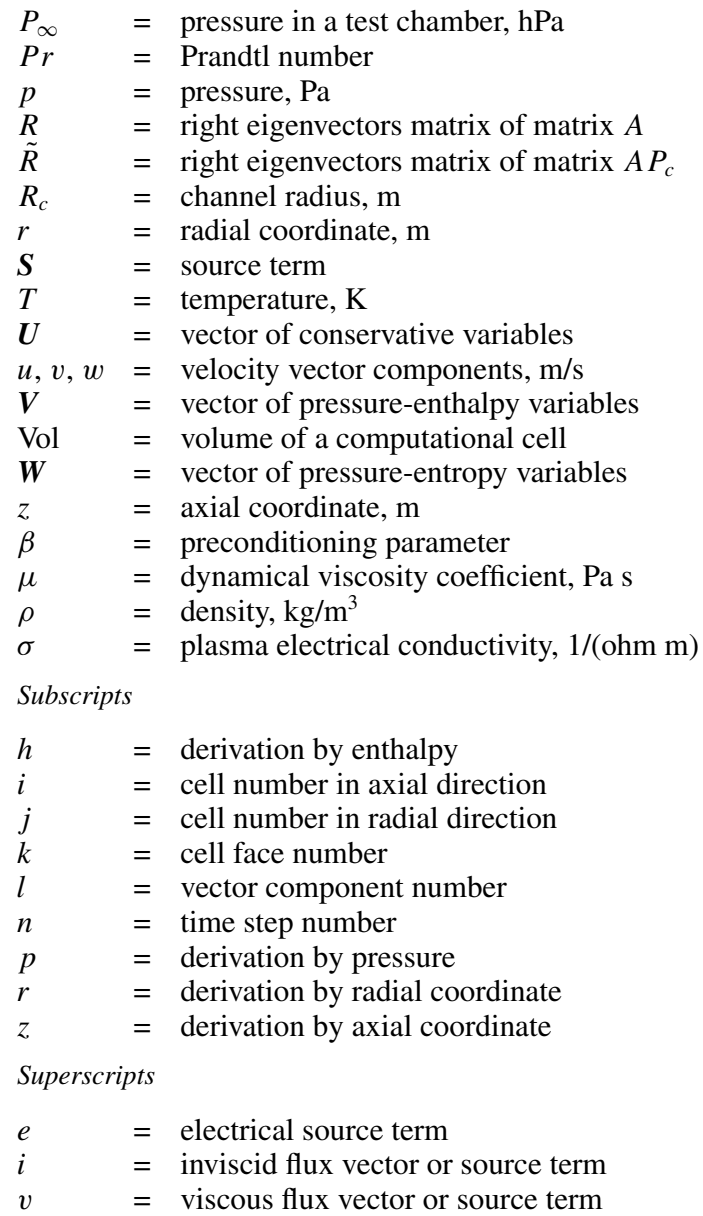

\section{Introduction}

A MODERN application of the inductively coupled plasmas is simulating thermochemical interaction of high-enthalpy gas 
flows with thermal protection materials (TPM) at the hypersonic flight conditions. ${ }^{1,2}$ The IPG plasmatrons at the Institute for Problems in Mechanics of the Russian Academy of Sciences (IPM RAS) appeared to be very efficient tools for the TPM aerothermal testing, and predicting the TPM catalycity. ${ }^{1}$ The efficient capabilities of the $100-\mathrm{kW}$ IPG-4 plasmatron for the simulation of physicochemical processes accompanying the hypersonic entry of a vehicle aeroshell in the Martian atmosphere have been recently demonstrated. ${ }^{3,4}$ But in fact, the potential capabilities of research and design facilities to simulate reacting flow physics and plasma/surface interaction can be revealed, if the measurements are properly combined with computational-fluid-dynamics (CFD) modeling. CFD modeling is an indispensable tool for aerothermal testing in order to carry out CFD codes validation, to rebuild flowfield in plasmatron, and to extract TPM catalycity, related to atom recombination, from heattransfer measurements. In general, the problem is rather tricky. Up to now there are few computations of the nonequilibrium air ${ }^{5}$ and $\operatorname{argon}^{6}$ plasma flows coupled with the rf electromagnetic field.

In prior papers ${ }^{4,7}$ the entire flowfield into plasmatron has been calculated separately in two, ${ }^{7}$ three, ${ }^{4}$ or several ${ }^{8}$ computational zones. Usually, plasma torch is the first zone, jet flow around the model is the second one, and boundary layer on the test model is the third one.

In the present paper a new numerical algorithm is presented, and computer codes have been developed to simulate the problem without dividing the entire domain into different subregions. The algorithm allows us to simulate both sub- and supersonic regimes in united manner.

In the paper we present the capabilities of CFD modeling inductively coupled plasma and some results of computations carried out in the wide range of the IPG-4 plasmatron operating conditions for equilibrium air plasma. The advanced technology developed for calculations of the plasma transport properties has been used. It is based on the rigorous modification of the Chapman-Enskog formalism ${ }^{9}$ and appropriate database for thermodynamic and transport properties.

A time-relaxation implicit method along with the implicit is used in the computer code developed. The code is based on a new approach to use a preconditioning technique with the total-variationdiminishing (TVD) schemes. ${ }^{10,11}$ It allows one to simulate both lowMach-number and supersonic flows in a common manner. In Ref. 11, the code used has been validated by a comparison against the computational results obtained in the von Kármán Institute (VKI) for Fluid Mechanics (Brussels) ${ }^{5,8,12}$ and the IPM RAS. ${ }^{4}$

Simulation of both underexpanded and overexpanded supersonic jets in the plasmatron has been carried out. A quite reasonable correspondence with the experimental data is observed.

\section{Thermochemical Equilibrium Model}

The IPG-4 plasmatron can operate in the wide range of pressure $10-10^{3} \mathrm{hPa}$ within a plasma torch. The relevant properties of the chemically reacting air plasma flow alter drastically in this pressure range: from strong thermal nonequilibrium conditions to local thermal equilibrium (LTE). Therefore, there is no single thermochemical model and appropriate CFD code that are applicable for all plasmatron operating conditions. A relative border between nonequilibrium and LTE inductively coupled subsonic air plasma flows for the IPG-4 facility we estimate as $50 \mathrm{hPa}$. In the discharge channel air plasma above this pressure can be considered under LTE conditions. Though, an underexpanded air jet after a sonic nozzle in the plasmatron is always under thermochemical nonequilibrium. Nevertheless, the thermochemical equilibrium approximation for the entire plasmatron flowfield appears to be quite important for the development and validation of the numerical methods and appropriate CFD codes in the wide range of operating conditions. Implementation of a nonequilibrium model is out of the scope of this paper and is left for further development. A comparison with experiments gives a qualitative indication that such a simplified model is not completely irrelevant, and the code can be used for a real applied configuration at least under the assumptions on the physical model used. The equilibrium model can be considered as a reference case if we study the influence of nonequilibrium chemistry on the locations of the shock waves. That reference equilibrium case can be useful for predicting the pressure field. Such a model can also give us an estimation of the heat flux at the stagnation point relevant to the ideal catalytic wall.

The applied gas dynamic and thermochemical model is quite traditional for inductively coupled plasma computations. ${ }^{13}$ We assume that a plasma flow in a cylindrical discharge channel and test chamber is a stationary, laminar, and axisymmetric one with a swirl in azimuth direction. In our studying, the high-frequency electromagnetic field does not influence plasma transport properties, radiative processes are neglected, and the flow is under LTE. The model assumes that the elemental composition of the plasma is uniform: the demixing effect is not taken into account. That allows us to use tabulated values for both the thermodynamic and transport properties. The IPG- 4 operates in a supersonic regime with air at the pressure within the plasma torch below $300 \mathrm{hPa}$. In such regimes the effects of radiation are negligible in the terms of gas dynamics and heat transfer because a hemispherical emissivity of air is less than $10^{-4}$ at the relevant air temperature $8000 \mathrm{~K}$. We use the Stokes hypothesis on the zero bulk viscosity although we are considering air with diatomic species and flow conditions at which compressibility is significant.

\section{Governing Equations}

The plasma flow is assumed to be governed by the Navier-Stokes equations coupled with the magnetohydrodynamics (MHD) equations for rf electromagnetic field. To apply a time-relaxation method, the gas dynamical subset of governing equations is written in the following nonstationary form:

$$
\frac{\partial \boldsymbol{U}}{\partial t}+\frac{\partial\left(\boldsymbol{E}^{i}-\boldsymbol{E}^{v}\right)}{\partial z}+\frac{\partial\left(\boldsymbol{G}^{i}-\boldsymbol{G}^{v}\right)}{\partial r}+\boldsymbol{S}^{i}-\boldsymbol{S}^{v}=\boldsymbol{S}^{e}
$$

where

$$
\begin{aligned}
& \boldsymbol{U}=\left[\begin{array}{c}
\rho \\
\rho u \\
\rho v \\
\rho w \\
E
\end{array}\right], \quad \boldsymbol{E}^{i}=\left[\begin{array}{c}
\rho u \\
\rho u^{2}+p \\
\rho u v \\
\rho u w \\
u(E+p)
\end{array}\right], \quad \boldsymbol{G}^{i}=\left[\begin{array}{c}
\rho v \\
\rho v u \\
\rho v^{2}+p \\
\rho v w \\
v(E+p)
\end{array}\right] \\
& \boldsymbol{E}^{v}=\left[\begin{array}{c}
0 \\
\tau_{\mathrm{zz}} \\
\tau_{\mathrm{zr}} \\
\tau_{z \theta} \\
\tau_{\mathrm{zz}} u+\tau_{\mathrm{zr}} v+ \\
\tau_{z \theta} w+\frac{\mu}{P r} h_{z}
\end{array}\right], \quad \boldsymbol{G}^{v}=\left[\begin{array}{c}
0 \\
\tau_{\mathrm{zr}} \\
\tau_{\mathrm{rr}} \\
\tau_{r \theta} \\
\tau_{\mathrm{zr}} u+\tau_{\mathrm{rr}} v+ \\
\tau_{r \theta} w+\frac{\mu}{P r} h_{r}
\end{array}\right] \\
& \boldsymbol{S}^{v}=\frac{1}{r}\left[\begin{array}{c}
0 \\
\tau_{\mathrm{zr}} \\
\tau_{\mathrm{rr}}-\tau_{\theta} \\
2 \tau_{r \theta} \\
\tau_{\mathrm{zr}} u+\tau_{\mathrm{rr}} v+\tau_{r \theta} w+\frac{\mu}{P r} h_{r}
\end{array}\right] \\
& \boldsymbol{S}^{i}=\frac{1}{r}\left[\begin{array}{c}
\rho v \\
\rho v u \\
\rho\left(v^{2}-w^{2}\right) \\
2 \rho v w \\
v(E+p)
\end{array}\right], \quad \boldsymbol{S}^{e}=\left[\begin{array}{c}
0 \\
0 \\
F^{l} \\
0 \\
Q
\end{array}\right] \\
& \tau_{\mathrm{zz}}=\frac{2}{3} \mu\left(2 \frac{\partial u}{\partial z}-\frac{\partial v}{\partial r}-\frac{v}{r}\right), \quad \tau_{\mathrm{rr}}=\frac{2}{3} \mu\left(2 \frac{\partial v}{\partial r}-\frac{\partial u}{\partial z}-\frac{v}{r}\right)
\end{aligned}
$$




$$
\begin{gathered}
\tau_{\theta \theta}=\frac{2}{3} \mu\left(2 \frac{v}{r}-\frac{\partial u}{\partial z}-\frac{\partial v}{\partial r}\right), \quad \tau_{z \theta}=\mu \frac{\partial w}{\partial z} \\
\tau_{\mathrm{zr}}=\mu\left(\frac{\partial u}{\partial r}+\frac{\partial v}{\partial z}\right), \quad \tau_{r \theta}=\mu\left(\frac{\partial w}{\partial r}-\frac{w}{r}\right) \\
E=\rho e+\rho \frac{u^{2}+v^{2}+w^{2}}{2}, \quad h=e+\frac{p}{\rho}
\end{gathered}
$$

The equations are given in dimensional form and written in cylindrical coordinates $(z, r)$ accounting for the tangential velocity component $w ; t$ is the time; $\tau_{\mathrm{zz}}, \tau_{\mathrm{zr}}, \tau_{z \theta}, \tau_{\mathrm{rr}}, \tau_{r \theta}, \tau_{\theta \theta}$ are the viscous stress tensor components corresponding to axial, radial, and tangential coordinates. Axial symmetry with nonzero transversal momentum is assumed. The axial component of the Lorentz force is neglected. The averaged Lorentz force radial component $F^{l}$ and Joule heating term $Q$ in the right-hand side of Eq. (1) are expressed in terms of the tangential component of the electric field complex amplitude $E_{\theta}$. The axial component of the Lorentz force is neglected (see the following).

The governing equations are closed by constitutive relations, which define electrical conductivity, transport properties, and equation of state of plasma under LTE. The equation of state, conductivity, and transport properties are handled in a table form.

The boundary conditions are as follows: necessary flow parameters besides the pressure are specified at annular inlet slot at the channel entry section; the pressure is set in the test chamber; velocity components equal zero at rigid surfaces; and symmetry conditions are applied at the axis.

\section{Numerical Method}

A structured grid that is adaptive to the flow geometry is used. All metric information is expressed in the terms of finite volume method notations. The second-order-upwind TVD scheme, originally developed by Yee et al., ${ }^{14}$ is used to approximate flux vectors and flux-vector Jacobian matrices at the cell faces. The scheme is based on local characteristic approach and has a second order of accuracy in space almost everywhere excepting extrema of the characteristic variables. The corresponding set of linear algebraic equations can be written in $\delta$ form as

$$
\begin{aligned}
\{\boldsymbol{I}+ & \left.\frac{\tau}{\mathrm{Vol}}\left[\sum_{k=1,4}\left\langle A-\frac{\partial \boldsymbol{F}_{n}^{v}}{\partial \boldsymbol{V}} \frac{\partial \boldsymbol{V}}{\partial \boldsymbol{U}}\right\rangle_{k}^{n}+\mathrm{Vol} \cdot\left(\frac{\partial \boldsymbol{S}^{i}}{\partial \boldsymbol{U}}-\frac{\partial \boldsymbol{S}^{v}}{\partial \boldsymbol{U}}\right)\right]\right\} \Delta \boldsymbol{U} \\
& =-\frac{\tau}{\mathrm{Vol}}\left[\sum_{k=1,4}\left\langle\boldsymbol{F}_{n}-\boldsymbol{F}_{n}^{v}\right\rangle_{k}^{n}+\mathrm{Vol} \cdot\left(\boldsymbol{S}^{i}-\boldsymbol{S}^{v}-\boldsymbol{S}^{e}\right)\right]
\end{aligned}
$$

where $\tau$ is the time step, index $k$ identifies one of cell faces $\left\{i-\frac{1}{2}, j\right.$; $\left.i+\frac{1}{2}, j ; i, j-\frac{1}{2} ; i, j+\frac{1}{2}\right\}$, brackets $\langle f\rangle_{k}^{n}$ denote numerical approximation of function $f$ at face $k, \boldsymbol{U}=(\rho, \rho u, \rho v, E)^{T}, \boldsymbol{V}=$ $(p, u, v, h)^{T}, \Delta \boldsymbol{U}=\boldsymbol{U}^{n+1}-\boldsymbol{U}^{n}, \boldsymbol{F}_{n}^{v}=n_{x} \boldsymbol{E}^{v}+n_{y} \boldsymbol{G}^{v}$.

Numerical inviscid flux vector at face $\left(i+\frac{1}{2}, j\right)$ is given by

$$
\begin{aligned}
& \phi_{i+\frac{1}{2}}^{l}=\frac{1}{2} \psi\left(a_{i+\frac{1}{2}}^{l}\right)\left(g_{i}^{l}+g_{i+1}^{l}\right)-\psi\left(a_{i+\frac{1}{2}}^{l}+\gamma_{i+\frac{1}{2}}^{l}\right) \alpha_{i+\frac{1}{2}}^{l} \\
& \left\langle\boldsymbol{F}_{n}\right\rangle_{i+\frac{1}{2}, j}^{n}=\frac{1}{2}\left[\boldsymbol{F}_{n}\left(\boldsymbol{n}_{i+\frac{1}{2}, j}, \boldsymbol{U}_{i, j}^{n}\right)+\boldsymbol{F}_{n}\left(\boldsymbol{n}_{i+\frac{1}{2}, j}, \boldsymbol{U}_{i+1, j}^{n}\right)\right. \\
& \left.\quad+R_{i+\frac{1}{2}, j} \cdot \Phi_{i+\frac{1}{2}, j}\right]
\end{aligned}
$$

The elements $\phi^{l}$ of the vector $\Phi$ for the upwind-TVD scheme are (the second index $j$ is omitted everywhere for simplicity):

$$
\phi_{i+\frac{1}{2}}^{l}=\frac{1}{2} \psi\left(a_{i+\frac{1}{2}}^{l}\right)\left(g_{i}^{l}+g_{i+1}^{l}\right)-\psi\left(a_{i+\frac{1}{2}}^{l}+\gamma_{i+\frac{1}{2}}^{l}\right) \alpha_{i+\frac{1}{2}}^{l}
$$

where $a_{i+1 / 2}^{l}$ is the eigenvalue of the matrix $A, \alpha_{i+1 / 2}^{l}=$ $R_{i+1 / 2}^{-1}\left(\boldsymbol{U}_{i+1}^{n}-\boldsymbol{U}_{i}^{n}\right), g_{i}^{l}=\min \bmod \left(\alpha_{i-1 / 2}^{l}, \alpha_{i+1 / 2}^{l}\right)$, and

$$
\gamma_{i+\frac{1}{2}}^{l}=\frac{1}{2} \psi\left(a_{i+\frac{1}{2}}^{l}\right) \begin{cases}\frac{\left(g_{i+1}^{l}-g_{i}^{l}\right)}{\alpha_{i+\frac{1}{2}}^{l}}, & \alpha_{i+\frac{1}{2}}^{l} \neq 0 \\ 0, & \alpha_{i+\frac{1}{2}}^{l}=0\end{cases}
$$

To satisfy the entropy condition, the numerical viscosity $\psi$ is corrected near sonic points as follows ${ }^{13}$ :

$$
\psi(z)= \begin{cases}|z|, & |z| \geq \varepsilon \\ \frac{\left(z^{2}+\varepsilon^{2}\right)}{2 \varepsilon}, & |z|<\varepsilon\end{cases}
$$

The approximation of the numerical flux Jacobian at the cell face $\left(i+\frac{1}{2}, j\right),\langle A\rangle_{i+1 / 2, j}^{n}$, is obtained after a linearization of the numerical flux term given by Eq. (3):

$$
\begin{aligned}
& \langle A\rangle_{i+\frac{1}{2}, j}^{n} \Delta \boldsymbol{U}_{i j}=\frac{1}{2}\left[A\left(\boldsymbol{n}_{i+\frac{1}{2}, j}, \boldsymbol{U}_{i, j}^{n}\right) \times \Delta \boldsymbol{U}_{i, j}\right. \\
& \left.\quad+A\left(\boldsymbol{n}_{i+\frac{1}{2}, j}, \boldsymbol{U}_{i+1, j}^{n}\right) \Delta \boldsymbol{U}_{i+1, j}+\Omega_{i+\frac{1}{2}, j}\left(\Delta \boldsymbol{U}_{i+1, j}-\Delta \boldsymbol{U}_{i, j}\right)\right] \\
& \Omega_{i+\frac{1}{2}, j}=R_{i+\frac{1}{2}, j} \operatorname{diag}\left[\beta^{l}-\psi\left(a^{l}+\gamma^{l}\right)\right]_{i+\frac{1}{2}, j} R_{i+\frac{1}{2}, j}^{-1}
\end{aligned}
$$

where

$$
\beta_{i+\frac{1}{2}, j}^{l}=\psi\left(a_{i+\frac{1}{2}, j}^{l}\right) \frac{\left(g_{i, j}^{l}+g_{i+1, j}^{l}\right)}{2 \alpha_{i+\frac{1}{2}, j}^{l}}
$$

Values $a_{i+1 / 2, j}^{l} R_{i+1 / 2, j}, R_{i+1 / 2, j}^{-1}$ are calculated by an averaging procedure of some kind.

The flux vector, representing fluxes of conservative variables $U$ through the cell face, is

$$
\boldsymbol{F}_{n}=\boldsymbol{F} \cdot \boldsymbol{n}=\left[\begin{array}{c}
\rho \theta \\
\rho \theta u+n_{x} p \\
\rho \theta v+n_{y} p \\
\rho \theta(E+p)
\end{array}\right], \quad \theta=\boldsymbol{V} \cdot \boldsymbol{n}=n_{x} u+n_{y} v
$$

The corresponding Jacobian matrix and its eigensystem decomposition for the case of normal gas are given in Ref. 11 .

Inside a plasmatron discharge channel the gas flow is characterized by low Mach numbers (usually, $0.01<M<1$ ). As is well known, the governing equations become stiff as the Mach number tends to zero, and thus, the convergence rate of the iteration procedure is slow. In recent years, a number of preconditioning methods have been developed by several authors to solve the problem. ${ }^{15}$ The preconditioning matrix ${ }^{15}$ has the simplest diagonal form $P=\operatorname{diag}\left[\beta^{2}, 1,1,1\right]$ in the variables pressure-entropy $\boldsymbol{W}=(p, u, v, S)^{T}$. Then, in the conservative variables the preconditioning matrix $\boldsymbol{U}=(\rho, \rho u, \rho v, E)^{T}$ is as follows:

$$
P_{c}=\frac{\partial \boldsymbol{U}}{\partial \boldsymbol{W}} P \frac{\partial \boldsymbol{W}}{\partial \boldsymbol{U}}=\frac{\partial \boldsymbol{U}}{\partial \boldsymbol{V}} \frac{\partial \boldsymbol{V}}{\partial \boldsymbol{W}} P \frac{\partial \boldsymbol{W}}{\partial \boldsymbol{V}} \frac{\partial \boldsymbol{V}}{\partial \boldsymbol{U}}
$$

One can obtain the preconditioning matrix in the conservative variables for a gas with an arbitrary equation of state:

$$
P_{c}=I+\left(\beta^{2}-1\right) \frac{\Gamma}{a^{2}}\left\|\begin{array}{cccc}
-\frac{\varphi^{2}}{\Gamma} & u & v & -1 \\
-\frac{\varphi^{2}}{\Gamma} u & u^{2} & v u & -u \\
-\frac{\varphi^{2}}{\Gamma} v & u v & v^{2} & -v \\
-\frac{\varphi^{2}}{\Gamma} R & u R & v R & -R
\end{array}\right\|
$$




$$
\begin{array}{cc}
\frac{\varphi^{2}}{\Gamma}=\left[\frac{1}{2}\left(u^{2}+v^{2}\right)-e-\left.\rho e_{\rho}\right|_{p}\right], & \Gamma=\frac{1}{\left.\rho e_{p}\right|_{\rho}} \\
R=(E+p) \rho^{-1},\left.\quad e_{p}\right|_{\rho}=\frac{1}{\left.p_{e}\right|_{\rho}}, & \left.e_{\rho}\right|_{p}=-\frac{\left.p_{\rho}\right|_{e}}{\left.p_{e}\right|_{\rho}} \\
a^{2}=\left.p_{\rho}\right|_{e}+\left.\frac{p}{\rho^{2}} p_{e}\right|_{\rho} &
\end{array}
$$

Instead of Eq. (2) we solve

$$
\begin{gathered}
\left\{P_{c}^{-1}+\frac{\tau}{\operatorname{Vol}}\left[\sum_{k=1,4}\left\langle A-\frac{\partial \boldsymbol{F}_{n}^{v}}{\partial \boldsymbol{U}}\right\rangle_{k}^{n}+\mathrm{Vol} \cdot\left(\frac{\partial \boldsymbol{S}^{i}}{\partial \boldsymbol{U}}-\frac{\partial \boldsymbol{S}^{v}}{\partial \boldsymbol{U}}\right)\right]\right\} \Delta \boldsymbol{U} \\
=-\frac{\tau}{\operatorname{Vol}}\left[\sum_{k=1,4}\left\langle\boldsymbol{F}_{n}-\boldsymbol{F}_{n}^{v}\right\rangle_{k}^{n}+\mathrm{Vol} \cdot\left(\boldsymbol{S}^{i}-\boldsymbol{S}^{v}-\boldsymbol{S}^{e}\right)\right]
\end{gathered}
$$

But a TVD approximation is constructed in Eq. (8) as for the system

$$
P_{c}^{-1} \frac{\partial \boldsymbol{U}}{\partial t}+\frac{\partial\left(\boldsymbol{E}^{i}-\boldsymbol{E}^{v}\right)}{\partial z}+\frac{\partial\left(\boldsymbol{G}^{i}-\boldsymbol{G}^{v}\right)}{\partial r}+\boldsymbol{S}^{i}-\boldsymbol{S}^{v}=\boldsymbol{S}^{e}
$$

Thus

$$
\begin{aligned}
& \left\langle\boldsymbol{F}_{n}\right\rangle_{i+\frac{1}{2}, j}^{n}=\frac{1}{2}\left[\boldsymbol{F}_{n}\left(\boldsymbol{n}_{i+\frac{1}{2}, j}, \boldsymbol{U}_{i, j}^{n}\right)+\boldsymbol{F}_{n}\left(\boldsymbol{n}_{i+\frac{1}{2}, j}, \boldsymbol{U}_{i+1, j}^{n}\right)\right. \\
& \left.\quad+P_{c_{i+\frac{1}{2}, j}}^{-1} \tilde{R}_{i+\frac{1}{2}, j} \cdot \tilde{\Phi}_{i+\frac{1}{2}, j}\right] \\
& \langle A\rangle_{i+\frac{1}{2}, j}^{n} \Delta \boldsymbol{U}_{i j}=\frac{1}{2}\left[A\left(\boldsymbol{n}_{i+\frac{1}{2}, j}, \boldsymbol{U}_{i, j}^{n}\right) \times \Delta \boldsymbol{U}_{i, j}+A\left(\boldsymbol{n}_{i+\frac{1}{2}, j}, \boldsymbol{U}_{i+1, j}^{n}\right)\right. \\
& \left.\quad \times \Delta \boldsymbol{U}_{i+1, j}+P_{c_{i+\frac{1}{2}, j}^{-1}} \tilde{\Omega}_{i+\frac{1}{2}, j}\left(\Delta \boldsymbol{U}_{i+1, j}-\Delta \boldsymbol{U}_{i, j}\right)\right] \\
& \tilde{\phi}_{i+\frac{1}{2}, j}^{l}=\frac{1}{2} \psi\left(\tilde{a}_{i+\frac{1}{2}, j}^{l}\right)\left(\tilde{g}_{i, j}^{l}+\tilde{g}_{i+1, j}^{l}\right)-\psi\left(\tilde{a}_{i+\frac{1}{2}, j}^{l}+\tilde{\gamma}_{i+\frac{1}{2}, j}^{l}\right) \tilde{\alpha}_{i+\frac{1}{2}, j}^{l} \\
& \tilde{\Omega}_{i+\frac{1}{2}, j}=\tilde{R}_{i+\frac{1}{2}, j} \operatorname{diag}\left[\tilde{\beta}^{l}-\psi\left(\tilde{a}^{l}+\tilde{\gamma}^{l}\right)\right]_{i+\frac{1}{2}, j} \cdot \tilde{R}_{i+\frac{1}{2}, j}^{-1}
\end{aligned}
$$

Here, the variables with the tilde are associated with the preconditioned flux Jacobian $P_{c} A$.

It is easy to see that this is a general way to combine preconditioning with a TVD approximation. Following this way, it is possible to deduce, in particularly, the preconditioning method ${ }^{15}$ developed for the Roe scheme of a first order of approximation.

In detail our approach is given in Ref. 11, where the results, obtained by the code, described are compared against the codes developed at the IPM and VKI. In major characteristics, including temperature profiles, the difference mainly is within a few percent. An illustration of the comparison results is given next.

\section{Discharge Electrodynamics}

Suppose that the oscillating external current in separate inductor circular coils produces a monochromatic electric field with the complex amplitude $\boldsymbol{E}(z, r)$. Following Ref. 13, we use the assumption for the tangential component of the electric field amplitude $E_{\theta}(z, r)$ : $\partial E_{\theta} / \partial z \ll \partial E_{\theta} / \partial r$, which leads to a quasi-one-dimensional approximation of the MHD equations:

$$
\begin{gathered}
\frac{\mathrm{d}}{\mathrm{d} r}\left[\frac{1}{r} \frac{\mathrm{d}}{\mathrm{d} r}\left(r E_{\theta}\right)\right]=-i \omega \mu_{0} \sigma E_{\theta} \\
i \omega \mu_{0} H_{z}=\frac{1}{r} \frac{\mathrm{d}}{\mathrm{d} r}\left(r E_{\theta}\right)
\end{gathered}
$$

Here $\omega$ is the circular frequency of the inductor current and electric field; $\omega=2 \pi f$. In this approximation the vortical electric field amplitude is described by the single nonzero component $E_{\theta}$, the magnetic field amplitude has the single nonzero component $H_{z}$, and the axial component of Lorentz force equals zero. This quasione-dimensional approximation is an essential simplification of the problem; it leads to a boundary-value problem for the ordinary differential equation just shown to determine $E_{\theta}(z, r)$; the $z$ coordinate is a parameter only in this equation and $E_{\theta}$ depends on $z$ owing to boundary conditions. To determine $E_{\theta}$, we use also the symmetry condition at the axis $E_{\theta}(z, 0)=0$ and the condition at the discharge channel wall ${ }^{16}$ as follows:

$$
r=R_{c}: \frac{1}{r} \frac{\mathrm{d}}{\mathrm{d} r}\left(r E_{\theta}\right)=i \omega \mu_{0} H_{\mathrm{zc} 0}(z)
$$

Here $H_{\mathrm{zc} 0}$ is the axial component of the magnetic field amplitude produced only by the inductor current outside the plasma flow, and $\mu_{0}$ is the vacuum magnetic permeance. $H_{\mathrm{zc} 0}$ is determined by the inductor current amplitude, which in turn is determined in numerical solution by the value of the total power input in plasma $N_{\mathrm{pl}}=\eta N_{\mathrm{ap}}$, where $N_{\text {ap }}$ and $\eta$ are the plasmatron anode power and efficiency obtained by experimental measurements.

A comparison between the two-dimensional MHD equation solutions and the quasi-one-dimensional approximation results given in Ref. 17 showed quite a good accuracy of this approximation in a wide range of operating frequencies for the plasma torch geometry under consideration.

\section{Calculation of Plasma Transport Properties}

To solve the Navier-Stokes and Maxwell equations, the following transport coefficients are necessary: the viscosity, thermal conductivity, and electrical conductivity. In our approach the transport coefficients were calculated in advance for the equilibrium air plasma flows as functions of the pressure and temperature. The transport properties were calculated by the precise formulas of the ChapmanEnskog method $^{9}$ in the temperature range $300 \leq T \leq 15,000 \mathrm{~K}$. The first nonzero approximation is rather accurate to calculate transport coefficients for neutral gases, but for ionized gases it can lead to $\sim 50 \%$ error. ${ }^{18}$ Our calculations for high temperatures were made with $\zeta=2$ for the viscosity and $\zeta=4$ for other transport coefficients to provide $5 \%$ accuracy; here $\zeta$ is the order of approximation by Sonine polynomials, and $\zeta$ means the number of terms in Sonine polynomial expansions of Boltzmann's equation solution that provides transposition in the Chapman-Enskog method. The formulas ${ }^{9}$ exploited for the transport properties are much more convenient for the calculations than the classic formulas ${ }^{17}$ of the ChapmanEnskog method because these contain the determinants of the order $N(\zeta-1) \times N(\zeta-1)$ instead of the order $N \zeta \times N \zeta$.

\section{Computational Results on Underexpanded and Overexpanded Supersonic Jets}

The underexpanded and overexpanded supersonic jets exhausted from the IPG-4 plasmatron through a sonic and Laval nozzle into the test chamber have been considered in the current research.

The principal scheme of the ICP torch with a sonic nozzle is given in Fig. 1 (Ref. 7). The outer inductor is represented by parallel current-currying rings, which are considered as infinitely thin. The nozzle at the end of the channel allows us to reach the sonic velocity for the flow. After the nozzle the underexpanded jet interacts with

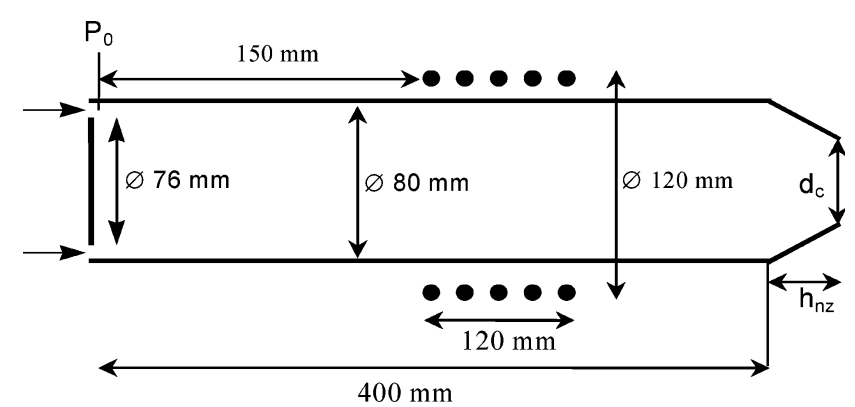

Fig. 1 Sketch of discharge channel with sonic nozzle. 


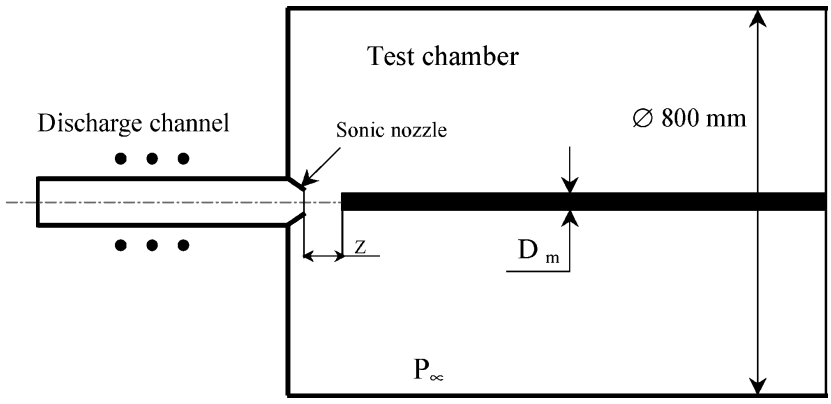

Fig. 2 Sketch of test chamber.

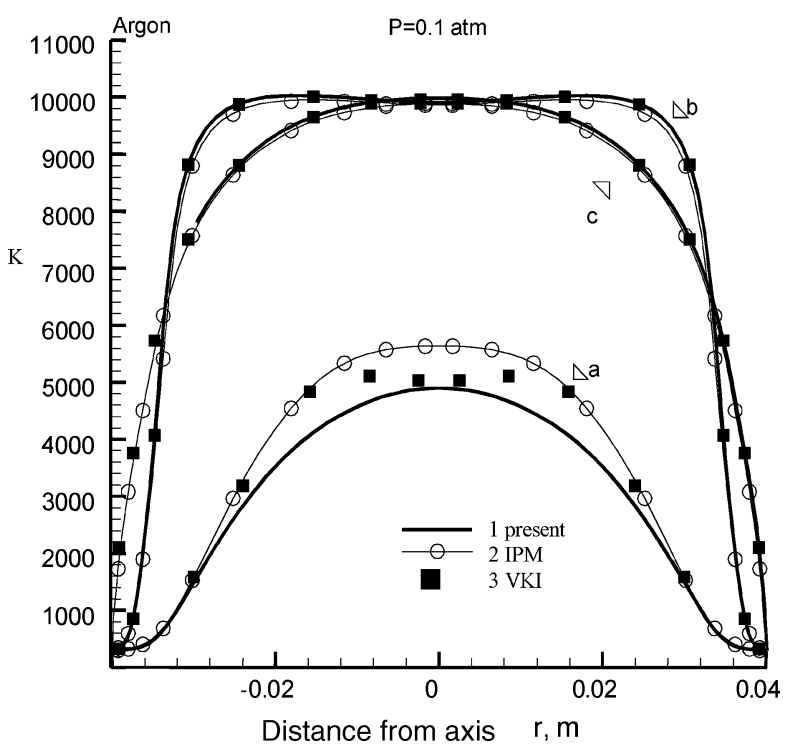

Fig. 3 Comparison of temperature profiles at $\mathrm{z}=$ a) 15 , b) 27, and c) $46 \mathrm{~cm}$ for argon.

a test sample located in the test chamber. The sketch of the test chamber is represented in Fig. 2.

The input parameters of the problem are as follows: gas is air, the angle of the flow spinning is $45 \mathrm{deg}$, the inductor frequency $f=1.76 \mathrm{MHz}$, and the temperature of the input gas and the temperature of the channel and chamber walls coincide and equal $300 \mathrm{~K}$.

A comparison of the results obtained by three absolutely different codes (code-to-code validation) was done for several operating pressures (1 atm; $0.1 \mathrm{~atm} ; 0.04 \mathrm{~atm}$ ) in the case of argon. For the comparison, we used the results obtained at the $\mathrm{IPM}^{7}$ and $\mathrm{VKI}^{17}$ using our own in-house well-tested codes. Temperature profiles are presented in Fig. 3 at the following axial positions in the plasmatron channel: $z=15 \mathrm{~cm}$ (inlet region), $z=27 \mathrm{~cm}$ (midcoil), and $z=46 \mathrm{~cm}$ (outlet). The results of our calculations (solid line) are compared against the IPM ${ }^{7}$ (circle-line) and $\mathrm{VKI}^{17}$ (squares) data. In this example the operating parameters used were as follows: the airflow rate $G=2.8 \mathrm{~g} / \mathrm{s}$, the pressure at the inlet slot $P_{0}=0.1 \mathrm{~atm}$, and the plasmatron anode power and its efficiency are $N_{\text {ap }}=23.5 \mathrm{~kW}$, $\eta=0.64$. The results obtained by all codes are in a reasonably good agreement. The relatively big difference in the inlet region is apparently explained by somewhat different numerical realization of the inlet boundary conditions. The most "full" temperature profile is observed in the midcoil section relative to the Joule heating area.

Numerical tests we have carried out ${ }^{11}$ reveal essential improvement in convergence rate if preconditioning technique (7-9) is used for low-Mach-number flows. The history of convergence shown in Fig. 4 corresponds to the calculation of channel flow with $M_{0}=10^{-2}$, where $M_{0}$ is the inlet Mach number. Because of an implicit scheme used, it is possible to integrate the governing equa-

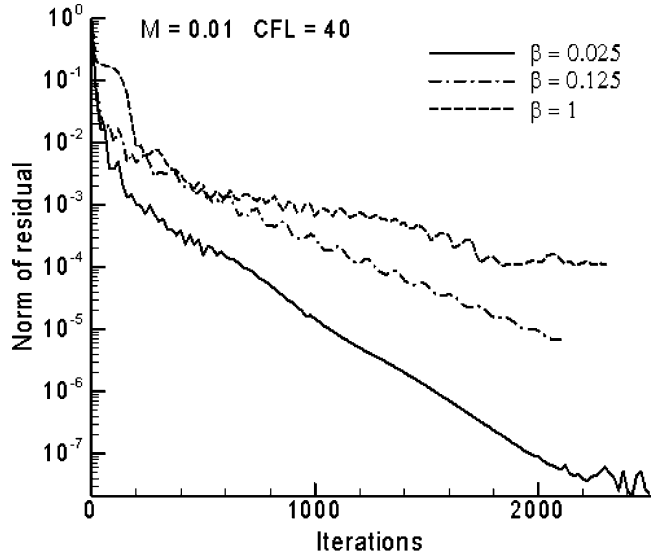

Fig. 4 Evolution of the norm of residual for different values of preconditioning parameter $\beta$.

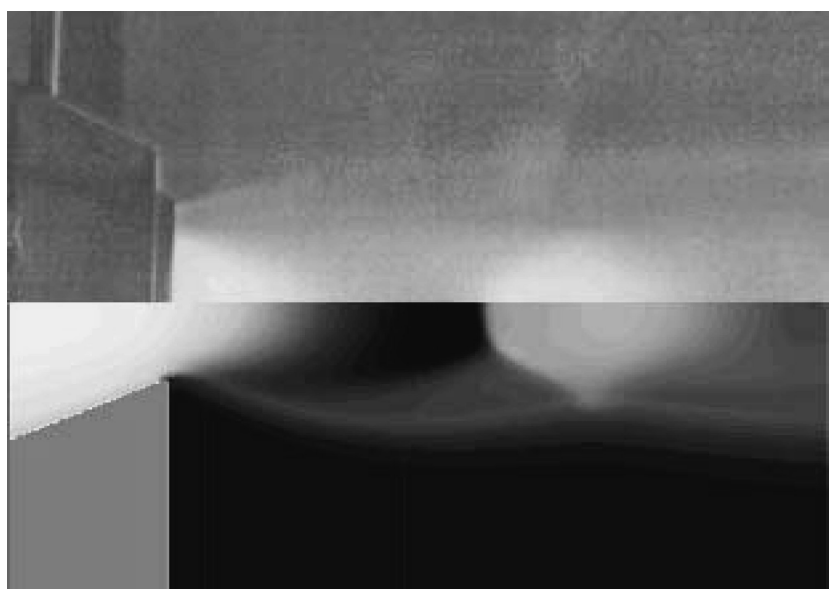

Fig. 5 Underexpanded supersonic plasma jet. The upper part is experiment; the lower one is shadow computational picture.

tions using high Courant-Friedrichs-Lewy numbers (CFL). In the calculations done, the CFL number equals 40. A linear system of Eqs. (8) was solved using the approximate factorization based on space splitting. The evolution of the residual norm is presented for different values of the preconditioning parameter $\beta$. The nonpreconditioning case corresponds to $\beta=1$. The final (limit) solution does not depend substantially on $\beta$ because of delta-form (8) used if the mesh is fine enough.

In the case of underexpanded jets, the following operation parameters are considered: $G=2.4 \mathrm{~g} / \mathrm{s}, N_{\text {ap }}=45 \mathrm{~kW}, \eta=0.64$, the sonic nozzle diameter $d_{c}=0.04 \mathrm{~m}$, and the nozzle length $h_{\mathrm{nz}}=0.045 \mathrm{~m}$. The pressure in the test chamber $P_{\infty}=6.4 \mathrm{hPa}$, whereas the pressure at the inlet slot is ${ }^{6} P_{0}=37.4 \mathrm{hPa}$. Strictly speaking, the last value is not necessary for the mathematical statement of the problem, and we will turn to this question later.

A comparison of the experimental ${ }^{7}$ and computational results is given in Fig. 5. The experimental photograph is shown in the upper part. For the comparison, the shadow contour plot picture of the internal energy is given in the lower part. The correspondence of the location of "casks," local compression shocks, and jet boundary is quite reasonable. We should remark here that first a comparison with the experiment used was done in Ref. 7 using another code and numerical approach. The correspondence with the experimental results obtained was satisfactory in terms of the stagnation point heat flux and pressure, but the problem was solved by considering flows in the channel and test chamber separately. Different codes and numerical methods were used to simulate these flow parts. In the second part (test chamber) the value $P_{0}$ set from the experiment was used as the boundary condition in Ref. 7. In our approach we solve the problem in a united manner, and $P_{0}$ is obtained in the 
Table 1 Pressure comparison between computational and experimental results

\begin{tabular}{llll}
\hline \hline & & \multicolumn{2}{c}{$P_{0}, \mathrm{hPa}$} \\
\cline { 3 - 4 }$N_{\text {ap }}, \mathrm{kW}$ & $d_{c}, \mathrm{~m}$ & Calc & Exp \\
\hline 52 & 0.024 & 51.8 & 50.4 \\
76 & 0.05 & 31.1 & 30.5 \\
\hline \hline
\end{tabular}

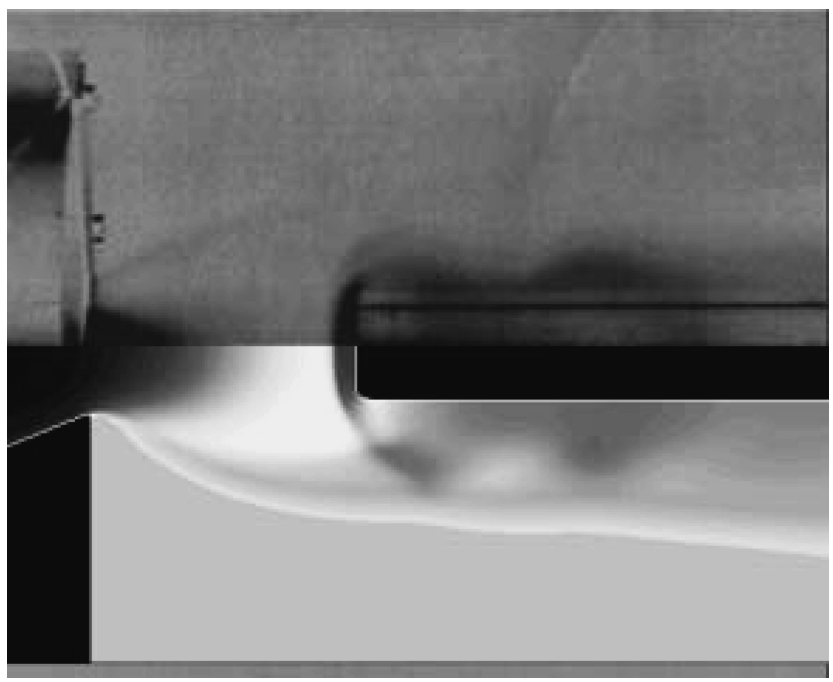

Fig. 6 Underexpanded supersonic plasma jet over sample cylinder. The upper part is experiment; the lower one is shadow computational picture.

calculations. In the present example we have $P_{0}=41 \mathrm{hPa}$, which gives us a $10 \%$ correspondence with the experimental result. The error of the pressure measurements in the experiments was within $10 \%$ (Refs. 4 and 19).

In the calculations, an adaptive mesh was used consisting of $100 \times 50$ nodes in the channel, $50 \times 50$ nodes in the nozzle domain, and $200 \times 200$ ones in the test chamber. The parameter $\beta$ is variable and has the order of the local Mach number.

An underexpanded jet and supersonic flow over a cylindrical sample, $2 \mathrm{~cm}$ in the diameter $D_{m}$, are shown in the Fig. 6 . The distance $z$ between the sonic nozzle and the cylinder equals $6 \mathrm{~cm}$. As in the preceding case, the experimental and computational results are presented in the figure. The locations of the shock wave before the cylinder, rarefaction, and compression waves quite coincide.

The correspondence between the calculated pressure $P_{0}$ and the experimental data at the other regimes investigated was even better. The comparison is given in Table 1 .

As was just mentioned, the multistep modeling of flow in a plasmatron ${ }^{7}$ consists of the calculation of the plasmatron channel, where the value $P_{0}$ is taken from the experiment, followed by the calculation of the nozzle and chamber domains. The comparison of our unified approach against a multistage one used in Ref. 7 shows the later technique might have an essential error somewhere as a result of the nozzle influence. In Figs. 7 and 8, the axial velocity component and temperature profiles, accordingly, are given at the end channel section under the same flow conditions as for Figs. 5 and 6 . The solid line corresponds to our approach, and the dashed one is the multistage technique.

Further, some examples of modeling overexpanded jets are given. In this case $G=2.8 \mathrm{~g} / \mathrm{s}, P_{0}=0.1 \mathrm{~atm}$, and $N_{\text {ap }}=31.3 \mathrm{~kW}$. On the subsonic outlet boundary of the test chamber, the pressure is set $13 \mathrm{hPa}$. Thus, rarefaction gas is created in the test chamber. Mach numbers are changed from $2 \times 10^{-2}$ in the circulated flow up to 2.5 at the nozzle exit. The parameters of the nozzle are as follows: the nozzle throat is $0.016 \mathrm{~m}$, the diameter of the exit section is $0.03 \mathrm{~m}$, and the half-angle of the supersonic part is $10^{\circ}$. In Fig. 9, the upper part corresponds to streamlines; the lower part is the enthalpy

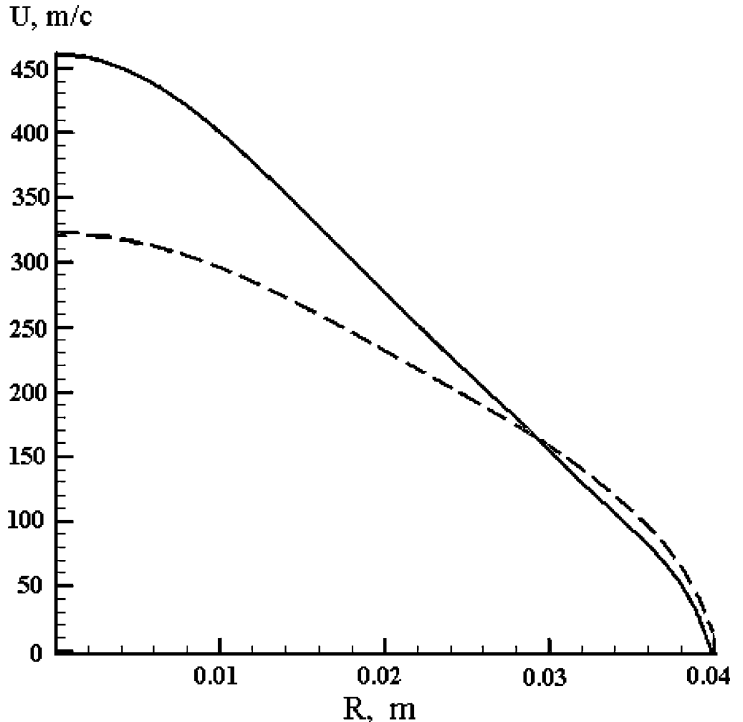

Fig. 7 Axial velocity profile at the channel end: - $\longrightarrow$, the unified calculation and ---, multistage approach. ${ }^{6}$

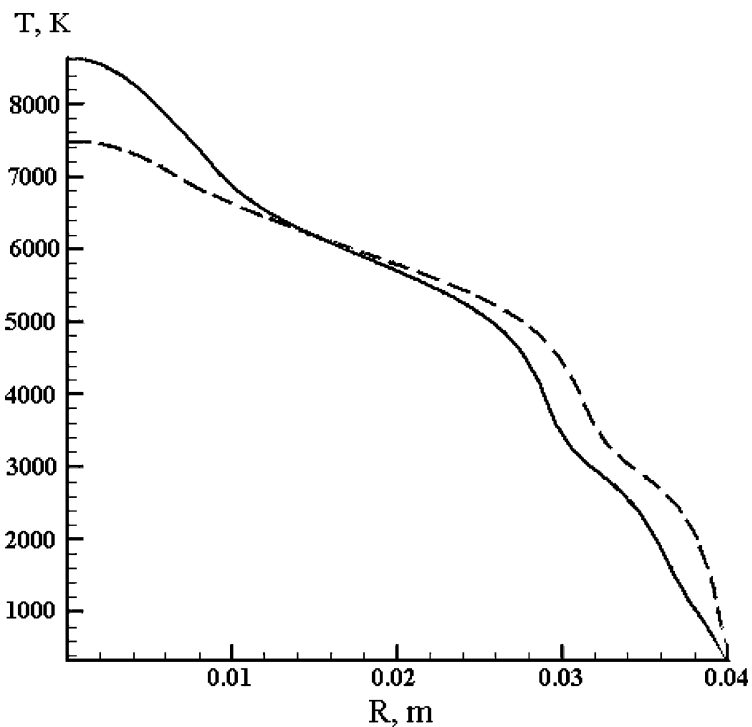

Fig. 8 Temperature profile at the channel end: - unified calculation and ---, multistage approach. ${ }^{6}$

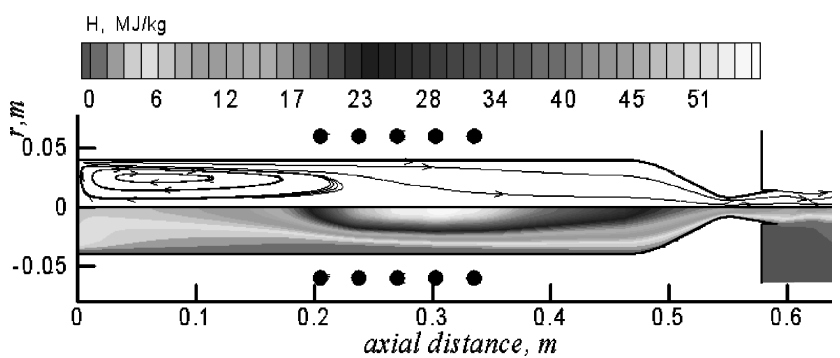

Fig. 9 Supersonic outflow from IPG-4 plasmatron. The upper part corresponds to streamlines and the lower one to enthalpy distribution.

distribution. The pressure is about $7 \mathrm{hPa}$ at the nozzle exit. Thus, the jet is overexpanded in this case, and the exhausted flow is accompanied by the typical system of shocks and rarefaction waves. A flow pattern near the nozzle, including the first two "casks," is presented in Fig. 10 by the distribution of pressure. The maximal velocity is about $3000 \mathrm{~m} / \mathrm{s}$ in this case. All calculations were performed by the same manner without dividing flowfield into different parts. 


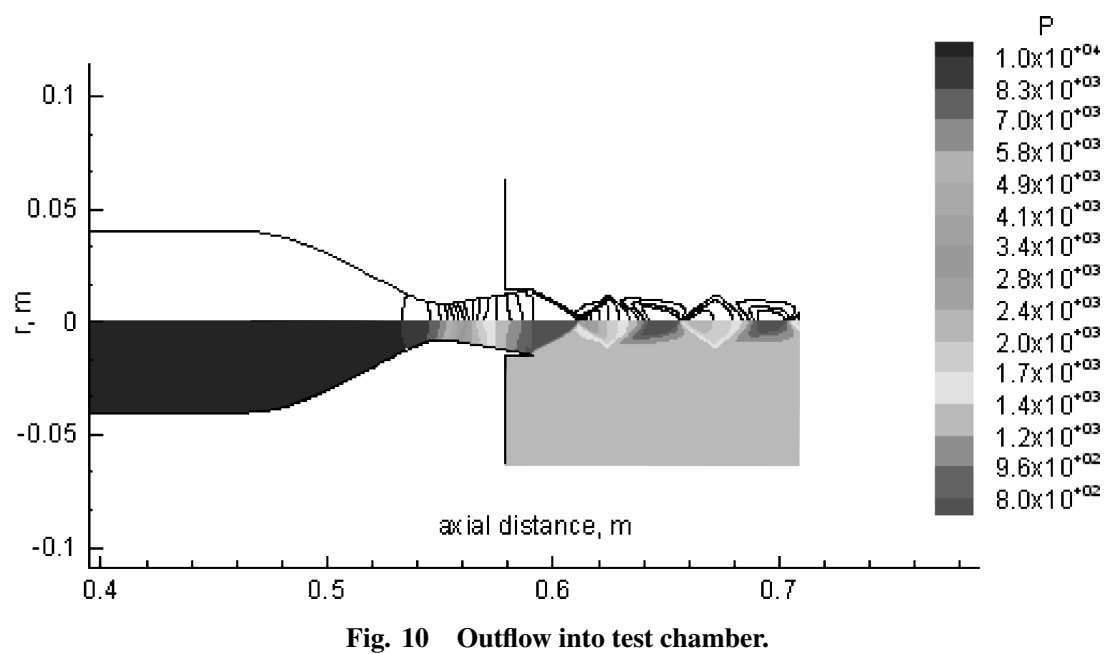

\section{Conclusions}

Numerical modeling of equilibrium inductive coupled plasma flows in a plasmatron has been done. An advanced technology developed for the calculations of the plasma transport coefficients based on the rigorous modification of the Chapman-Enskog formalism and appropriate database for thermodynamic and transport properties has been used.

A new effective preconditioning technique along with an implicit total-variation-diminishing scheme has been developed to solve the Navier-Stokes equations in both subsonic and supersonic regimes in a uniform manner. Numerical simulation of underexpanded and overexpanded supersonic jets, including the interaction with a sample in the test chamber, has been performed without dividing the domain investigated into different parts.

The computational results have been validated by a comparison of the flowfield structures with the experimental ones.

\section{Acknowledgments}

This research has been supported by the INTAS and ESA (Grant INTAS-ESA 99-01473). The authors are grateful to the referees for useful remarks.

\section{References}

${ }^{1}$ Gordeev, A. H., Kolesnikov, A. F., and Yakushin, M. I., "An Induction Plasmatron Application to 'Buran's' Heat Protection Tiles Ground Tests," Journal SAMPE, Vol. 28, No. 3, 1992, pp. 29-33.

${ }^{2}$ Bottin, B., Carbonaro, M., Van Der Haegen, V., and Paris, S., "Predicted and Measured Capability of the VKI 1.2 MW Plasmatron Regarding ReEntry Simulation," 3rd European Symposium on Aerothermodynamics for Space Vehicles, ESA SP-426, ESTEC, Noordwijk, The Netherlands, 1999, pp. 553-560.

${ }^{3}$ Kolesnikov, A. F., Yakushin, M. I., Pershin, I. S., and Vasil'evskii, S. A., "Heat Transfer Simulation and Surface Catalycity Prediction at the Martian Atmosphere Entry Conditions," AIAA Paper 99-4892, Nov. 1999.

${ }^{4}$ Kolesnikov, A. F., Pershin, I. S., Vasil'evskii, S. V., and Yakushin, M. I., "Study of Quartz Surface Catalycity in Dissociated Carbon Dioxide Subsonic Flows," Journal of Spacecraft and Rockets, Vol. 37, No. 5, 2000, pp. 573-579.

${ }^{5}$ Vanden Abeele, D., and Degrez, G., "Numerical Model of High-Pressure Air Inductive Plasmas Under Thermal and Chemical Nonequilibrium," AIAA Paper 2000-2416, June 2000.

${ }^{6}$ Selezneva, S. E., Sember, V., Gravelle, D. V., and Boulos, M. I., "Spectroscopic Validation of the Supersonic Plasma Jet Model," Journal of Physics D: Applied Physics, Vol. 35, No. 12, 2002, pp. 1338-1349.

${ }^{7}$ Afonina, N. E., Vasil'evskii, S. A., Gromov, V. G., Kolesnikov, A. F.,
Pershin, I. S., Saharov, V. I., Fateeva, E. I., and Yakushin, M. I., "Flow and Heat Transfer in Under-Expanded Air Jet Elapsing from Plasmatron Sonic Jet. Experiment and Numerical Simulation," Inst. for Problems in Mechanics, Russian Academy of Sciences, Moscow, 2001, Preprint 672, pp. 1-51 (in Russian).

${ }^{8}$ Magin, T., Vanden Abeele, D. P., and Degrez, G, "An Implicit Multiblock Solver for Inductive Plasma Flows," AIAA Paper 2000-2480, 2000

${ }^{9}$ Kolesnikov, A. F., and Tirskii, G. A., "Equations of Hydrodynamics for Partially Ionized Multi-Component Mixtures of Gases, Employing Higher Approximations of Transport Coefficients," Journal Fluid MechanicsSoviet Research, Vol. 13, No. 4, 1985, pp. 70-97.

${ }^{10}$ Vasil'evskii, S. A., Kolesnikov, A. F., Utyuzhnikov, S. V., and Sarma, G. S. R., "Physico-Chemical Data Base and Computation Results for Inductive Plasma Flows," Progress in Plasma Processing of Materials, edited by P. Fauchais and J. Amouroux, Begell House, New York, 1999, pp. 277-284.

${ }^{11}$ Utyuzhnikov, S. V., Konyukhov, A. F., Rudenko, D. V., Vasil'evskii S. A., Kolesnikov, A. F., and Sarma, G. S. R., "Numerical Modelling Subsonic and Supersonic Inductive Plasma Flows," European Congress on Computational Methods in Applied Sciences and Engineering, Paper 71, Sept. 2001.

${ }^{12}$ Vanden Abelee, D., and Degrez, G., "Efficient Computational Model for Inductive Plasma Flows," AIAA Journal, Vol. 38, No. 2, 2000, pp. 234-241.

${ }^{13}$ Boulos, M. I., "The Inductively Coupled Radio-Frequency Plasma," Journal of Pure and Applied Chemistry, Vol. 57, No. 9, 1985, pp. 1321-1352.

${ }^{14}$ Yee, H. C., Warming, R. F., and Harten, A., "Implicit Total Variation Diminishing Schemes for Steady-State Calculations," Journal of Computational Physics, Vol. 57, No. 3, 1985, pp. 327-360.

${ }^{15}$ Viozat, C., "Implicit Upwind Schemes for Low Mach Number Compressible Flows," INRIA, Technical Rept. 3084, Sophia-Antipolis, France, Jan. 1997.

${ }^{16}$ Vasil'evskii, S. A., Kolesnikov, A. F., and Yakushin, M. I., "Mathematical Models for Plasma and Gas Flows in Induction Plasmatrons," Molecular Physics and Hypersonic Flows, edited by M. Capitelli, NATO ASI Series, Kluwer, Dordrecht, The Netherlands, 1996, pp. 495-504.

${ }^{17}$ Vanden Abeele, D., Vasil'evskii, S. A., Kolesnikov, A. F., Degrez, G., and Bottin, B., "Code-to-Code Validation of Inductive Plasma Computations," Progress in Plasma Processing of Materials, edited by P. Fauchais and J. Amouroux, Begell House, New York, 1999, pp. 245-250.

${ }^{18}$ Capitelly, M., and Devoto, R. "Transport Coefficients of High Temperature Nitrogen," Physics of Fluids, Vol. 16, No. 11, 1973, pp. 1835-1841.

${ }^{19}$ Bykova, N. G., Vasil'evskii, S. A., Gordeev, A. N., Kolesnikov, A. F., Pershin, I. S., and Yakushin, M. I., "Determination of the Effective Probabilities of Catalytic Reactions on the Surfaces of Heat Shield Materials in Dissociated Carbon Dioxide Flows," Journal Fluid Dynamics, Vol. 32, No. 6, 1997, pp. 876-886.

C. Kaplan Associate Editor 